\title{
Beitrag des Brotes zur Bedarfsdeckung der schweizerischen Bevölkerung mit einigen wichtigen Nährstoffen
}

Contribution of Bread towards Covering Requirements of the Swiss Population with some essential Nutrients

\section{A. Blumenthal, P. Scheffeldt, R. Schönhauser}

INSTITUT FUR ERNÄHRUNGSFORSCHUNG DER STIFTUNG "IM GRUENE", SEESTR, 72, CH-8803 RUSCHLIKON, SCHWEIZ

GEGENWÄRTIGE SITUATION:

DIE MEHRHEIT DER SCHWEIZERISCHEN BEVÖLKERUNG BETRACHTET BROT ALS EINES DER WICHTIGSTEN NAHRUNGSMITTEL; DENNOCH IST DER KONSUM IM LAUFE DER ZEIT STARK GESUNKEN UND HAT SICH GEGENWÄRTIG BEI 130 G PRO KOPF UND TAG FUR 20 - 50 JÄHRIGE ERWACHSENE STABILISIERT.

VERTEILUNG DES BROTKONSUMS IN DER SCHWEIZ AUF DIE VERSCHIEDENEN BROTSORTEN

\begin{tabular}{|l|c|c|}
\hline \multicolumn{1}{|c|}{ BROTSORTE } & $\begin{array}{c}\text { ANTEIL } \\
G\end{array}$ & $\begin{array}{c}\text { ANTEIL } \\
\%\end{array}$ \\
\hline RUCHBROTE & 53.5 & 41 \\
HALBWEISSBROTE & 41.5 & 32 \\
WEISSBROTE & 15.5 & 12 \\
SPEZIALBROTE & 19.5 & 15 \\
(AUS HOCHAUSGE- & & \\
MAHLENEN MEHLEN) & & \\
\hline TOTAL & $130 \mathrm{G}$ & $100 \%$ \\
\hline
\end{tabular}

BEDARFSDECKUNG IN \% DURCH 130 G JE BROTSORTE NACH DEN EMPFEHLUNGEN DER DGE (MÄNNER)

\begin{tabular}{|l|c|c|c|}
\hline BROTSORTE & VITAMIN B1 & VITAMIN B & NIACIN \\
\hline RUCHBROT & 16.5 & 5.0 & $11.5-19.5$ \\
HALBWEISSBROT & 8,0 & 3.5 & $7.5-12.5$ \\
$\begin{array}{l}\text { REVITAMINISIIER- } \\
\text { TES HALBWEISS- } \\
\text { BROT }\end{array}$ & 17.0 & 9.0 & $22.5-37.5$ \\
$\begin{array}{l}\text { WEISSBROT } \\
\text { REVITAMINISIER- }\end{array}$ & 3.5 & 2.5 & $6-10$ \\
TES WEISSBROT & 21.5 & 12.5 & $27-44$ \\
\hline
\end{tabular}

DURCHSCHNITTLICHE ZUFUHR AN VITAMINEN DURCH 130 G BROT UNTER BERUCKSICHTIGUNG DER VERZEHRSGEWOHNHEITEN

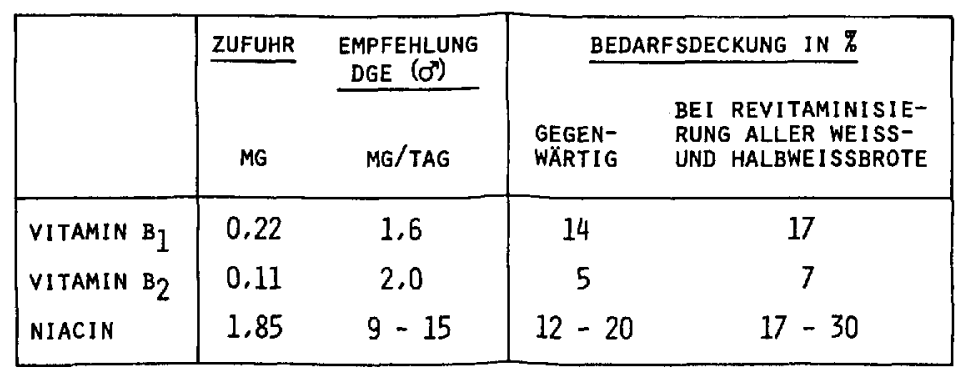

SCHLUSSFOLGERUNG:

1. BROT DECKT $12,5 \%$ DES BEDARFS AN ENERGIE: DIE BEDARFSDECKUNG AN THIAMIN, NIACIN, PHOSPHOR, EISEN UND MANGAN LIEGT Z,T. WESENTLICH HÖHER.

2. DIE NATRIUMAUFNAHME DURCH DAS BROT IST ALS ZU HOCH EINZUSTUFEN.

3. RUND $21 \%$ DES BALLASTSTOFFVERZEHRS ENTFALLEN AUF BROT.

4. DIE REYITAMINIERUNG ALLER HALBWEISS- UND WEISSBROTE WÜRDE ZU EINER MARKANTEN ERHÖHUNG DER VERSORGUNG BEI DEM GROSSEN PERSONENKREIS FUHREN, DER WEITGEHEND ODER AUSSCHLIESSLICH DIESE BROTSORTEN KONSUMIERT,

EMPFEHLUNGEN :

1. VERMEHRTE REVITAMINIERUNG VON WEISS- UND HALBWEISSBROTEN.

2. ANREIZE ZUM VERMEHRTEN KONSUM VON BROTEN AUS HOHER AUSGEMAHLENEN MEHLEN.

3. VERBESSERUNG DER PRODUKTIONSMETHODEN IM HINBLICK AUF GERINGERE VITAMINZERSTÖRUNG, REDUKTION DES KOCHSALZ- UND PHYTINSÄUREGEHALTES IN KONSUMFERTIGEN BROTEN. 